\title{
New Assembling-Disassembling Technology Using Electromagnetically Active Adhesives-Dielectric Behaviour
}

\author{
Vasilica Alina Neamtu, Ramona Burlacu, Cristina Bratescu, and Stefan Ursache
}

\begin{abstract}
The work refers to a new assembling disassembling technology based on designing functionally graded bonds by incorporating in the adhesive matrix electromagnetically active nanofillers. These kid of modified adhesives applied with various concentrations of nanofillers within the joints, can significantly increase the functionality of the structure when they are activated in electromagnetic field, at different frequencies. In the same electromagnetic field, the insertion of nanoparticles within the joint provides an immediate disassembly mechanism. The electromagnetic field can be switched using specific triggers. We have investigated the dielectric behavior of modified adhesives and the matrix we used was HF2030 with different percent of nanoparticles.
\end{abstract}

Index Terms-Dielectric measurements, magnetic nanoparticles, modified adhesives, nanostructured adhesives.

\section{INTRODUCTION}

Important components with potentially good chances of reuse, such as plastic and composite modules are bonded nowadays during the manufacturing processes with mechanical fasteners, welding or adhesives methods. The biggest disadvantage of the first two is that they are not reversible. The financial interest for recycling and reusing the high value components and the innovative solutions for reversibly disassembly are of great importance and interest for many of the manufacturing industries [1].

The insertion of nanofillers provides new functional properties to the modified adhesives, as well as improvement of mechanical performances, helpful for the adhesive assembling process. The new bonding process, activated in electromagnetic field, offers advantages as: simplification of productive lay-out, process reversibility and rapidity [2].

Measuring dielectric properties of materials in the frequency fields [3], is of great importance especially in the fields of research such as materials science, design of microwave circuits, developing adsorbents, biological research, etc. The materials dielectric behavior is important because it can provide electrical and magnetic characteristics

Manuscript received December 5, 2012; revised February 10, 2013. This work was supported in part by the Manunet Eranet Magbond 7-042/2011 project financed by UEFISCDI and the project PERFORM-ERA "Postdoctoral Performance for Integration in the European Research Area" (ID-57649), financed by the European Social Fund and the Romanian Government.

The authors are with the "Gheorghe Asachi" Technical University of Iași, Romania, Faculty of Electrical Engineering (e-mail: sml_alinutza@yahoo.com,rburlacu@ee.tuiasi.ro, cbratescu@ee.tuiasi.ro, sursache@ee.tuiasi.ro). of materials, matter extremely useful in many areas of research and development [4].

To achieve a complete characterization of the material and their electromagnetic performance, research should focus both on the experimental measurements and numerical analysis. The final electromagnetic properties will depend on microscopic structure and intrinsic properties of the material used to implement this structure. Also the use of magnetic nanoparticles [5], within the adhesives matrices can have a significant and unpredictable result.

\section{NANOPARTICLES ANALYSIS}

The samples were prepared in Vaber Industriale R\&D Laboratories.

Some of the particles we chose for the experimental activity are presented as it follows:

\section{A. Cobalt ferrite Nanoparticles (CoFe2O4) - Nanotesla [6]}

Spherical in shape with a narrow particle size distribution 5 $-20 \mathrm{~nm}( \pm 10 \%$ st.d. $)$ and specific surface area in the $80-130$ $\mathrm{m} 2 / \mathrm{g}$ ( $\pm 3 \%$ st.d.) range (Fig. 1$)$.

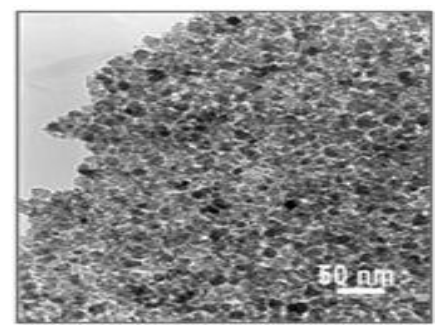

Fig. 1. TEM image of Co-ferrite nanoparticles

\section{B. Maghemite Nanoparticles ( $\gamma$-Fe2O3) - Nanotesla}

Spherical in shape with a narrow particle size distribution typically $8-20 \mathrm{~nm}( \pm 10 \%$ st.d.) and specific surface area in the $80-130 \mathrm{~m} 2 / \mathrm{g}$ ( $\pm 3 \%$ st.d.) range (Fig. 2).

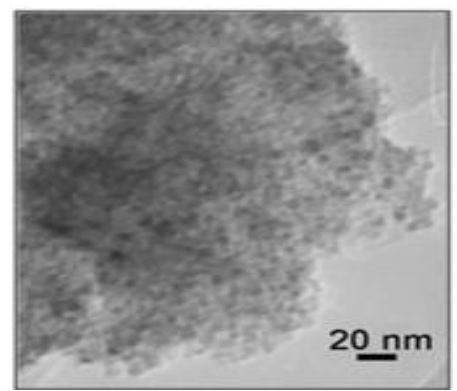

Fig. 2. TEM image of maghemite $(\gamma$-Fe2O3) nanoparticles 


\section{Manganese Zinc Ferrite Nanoparticles}

$(M n x Z n y F e(3-x-y) O 4 ; x+y=1)$ - Nanotesla

Relatively spherical in shape with an average particle size of around $5-30 \mathrm{~nm}( \pm 10 \%$ st.d.) and specific surface area in the $80-130 \mathrm{~m} 2 / \mathrm{g}( \pm 3 \%$ st.d.) range (Fig. 3).

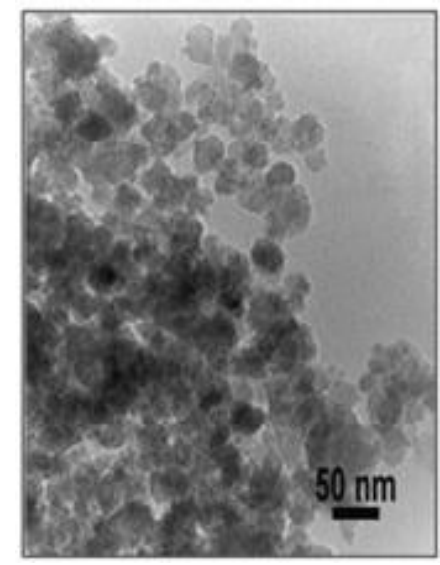

Fig. 3. TEM image of MnZn-ferrite nanoparticles

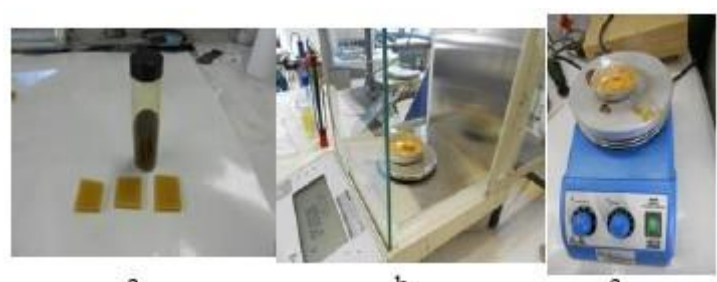

a.

b.

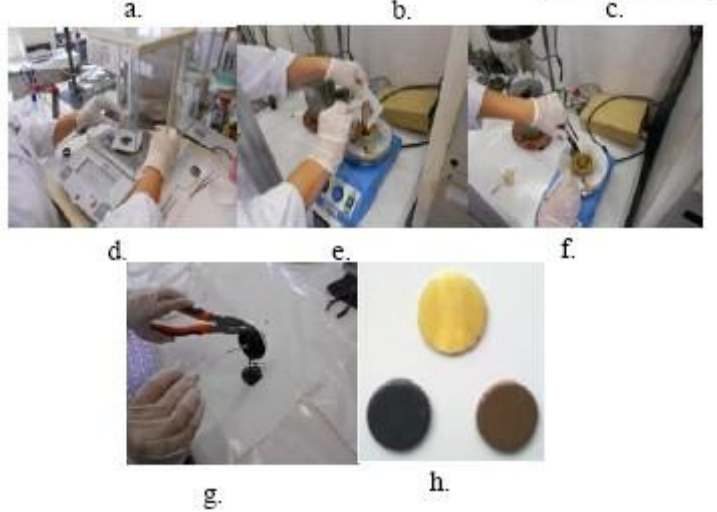

Fig. 4. a. Adhesive matrix (at room temperature) and the nanoparticles, b. Weighing the adhesive, c. Melting the adhesive around $250^{\circ} \mathrm{C}$, d. Weighing the nanoparticles, e. Adding particles to molten adhesive, f. Mixing, g. Nanofilled adhesive cools down, h. Adhesive matrix and 2 samples of nanofilled adhesive.

\section{MiXING THE ADHESIVE WITH NANOPARTICLES}

We have manually mixt a small quantity of nanoparticles (magnetite $\mathrm{Fe}_{2} \mathrm{O}_{3}$ ) with the compounded adhesive matrix, MAG HF 2030, in a molten state. In Fig. 4 it can be observed the procedure to obtain relevant samples of nanoactivated adhesive filled with 2-5-8\% in weight of nanoparticles.

We used the adhesive matrix HF2030 to investigate the dielectric properties behavior of nanofilled adhesives.

\section{Dielectric Properties - RESUlts}

We have studied the use of dielectric spectroscopy [7] for technological analysis of knowledge based composite materials, with target on their potential appliances The dielectric properties of the nanostructured adhesives that we have investigated are: dielectric constant $\left(\varepsilon^{\prime}\right)$ and loss tangent $(\tan \delta)$ and were determined using a Broadband Dielectric Spectrometer (Novocontrol GMBH) [8], who works in a wide frequency range $(10 \mu \mathrm{Hz} \div 8 \mathrm{GHz})$ and a wide temperature range $\left(-160^{\circ} \mathrm{C} \div+400{ }^{\circ} \mathrm{C}\right)$.

The samples materials were sandwiched between two 20 $\mathrm{mm}$ gold plated electrodes forming a sample capacitor, and tested within ZGS Alpha Active Sample Cell. The test temperatures were between $\left(35 \div 90{ }^{\circ} \mathrm{C}\right)$. The temperature was increased gradually with a step of 5 Celsius degrees (the temperature stabilization time $=3$ minutes). The AC voltage applied to the capacitor was equal to $1 \mathrm{~V}$. Temperature was controlled using a nitrogen gas cryostat and the temperature stability of the sample was better than $0.1{ }^{\circ} \mathrm{C}$.

\section{A. Dielectric Constant $\left(\varepsilon^{\prime}\right)$}

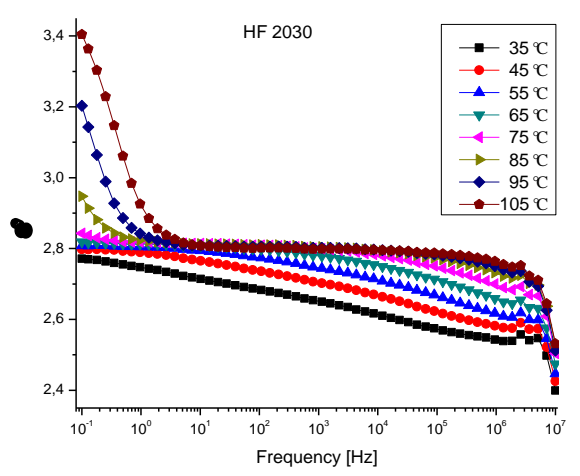

Fig. 5. Frequency dependencies of dielectric constant, $\varepsilon^{\prime}$, corresponding to the adhesive matrix HF2030

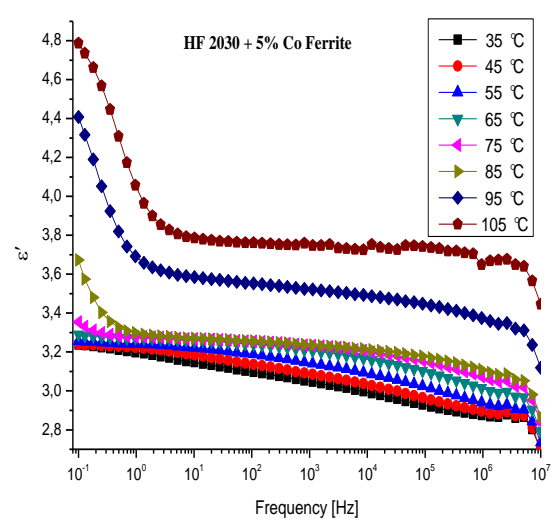

Fig. 6. Frequency dependencies of dielectric constant, $\varepsilon^{\prime}$, corresponding to the adhesive matrix HF2030 $+5 \%$ CoFerrite

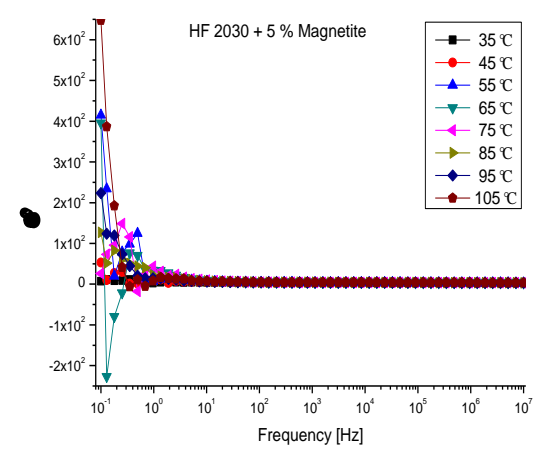

Fig. 7. Frequency dependencies of dielectric constant, $\varepsilon^{\prime}$, corresponding to the adhesive matrix HF2030 $+5 \%$ Magnetite 


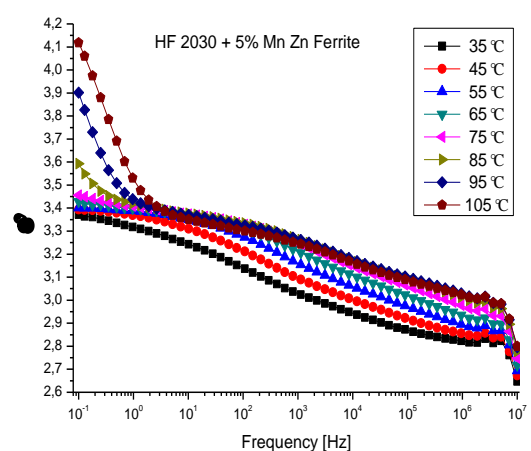

Fig. 8. Frequency dependencies of dielectric constant, $\varepsilon^{\prime}$, corresponding to the adhesive matrix HF2030 + 5\% MnZnFerrite

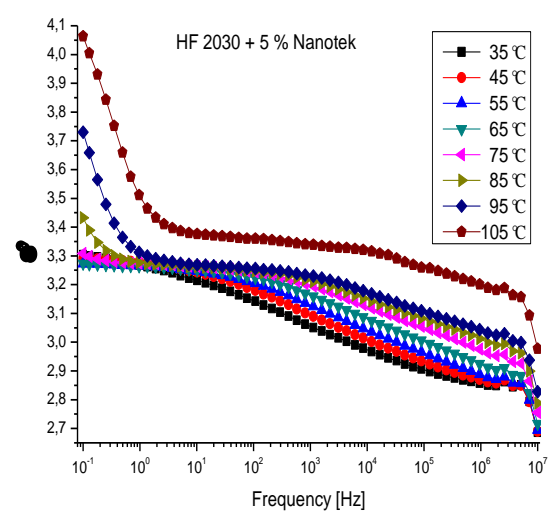

Fig. 9. Frequency dependencies of dielectric constant, $\varepsilon^{\prime}$, corresponding to the adhesive matrix HF2030 + 5\% Nanotek

Fig. 5 shows the frequency dependencies of dielectric constant, $\varepsilon^{\prime}$, corresponding to the adhesive matrix HF2030 and Fig. 6-9 show the frequency dependencies of dielectric constant, $\varepsilon^{\prime}$, corresponding to the adhesive matrix with $5 \%$ of various nanoparticles. The value of $\varepsilon^{\prime}$ decreased when increasing the frequency, which indicates that the major contribution to the polarization comes from orientation polarization. The decrease of $\varepsilon^{\prime}$ with increasing frequency represents a normal behavior in most dielectric materials. This is due to dielectric relaxation which is the cause of anomalous dispersion. From a structural point of view, the dielectric relaxation involves the orientation polarization which in turn depends upon the molecular arrangement of dielectric material [9]. The dielectric constant practically doesn't change around melting point of composites for the entire domain of frequencies $10^{-1}-10^{7} \mathrm{~Hz}$

\section{B. Loss Tangent $(\operatorname{tg} \delta)$}

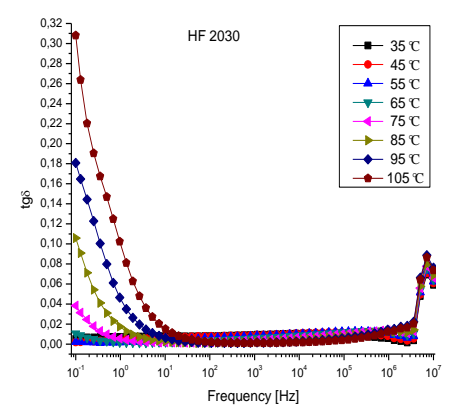

Fig. 10. Frequency dependencies of loss tangent, $\operatorname{tg} \delta$, corresponding to HF 2030

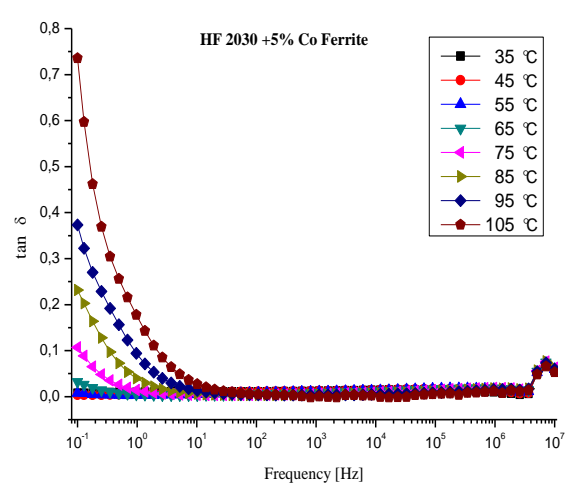

Fig. 11. Frequency dependencies of loss tangent, $\operatorname{tg} \delta$, corresponding to HF $2030+5 \%$ Co Ferrite

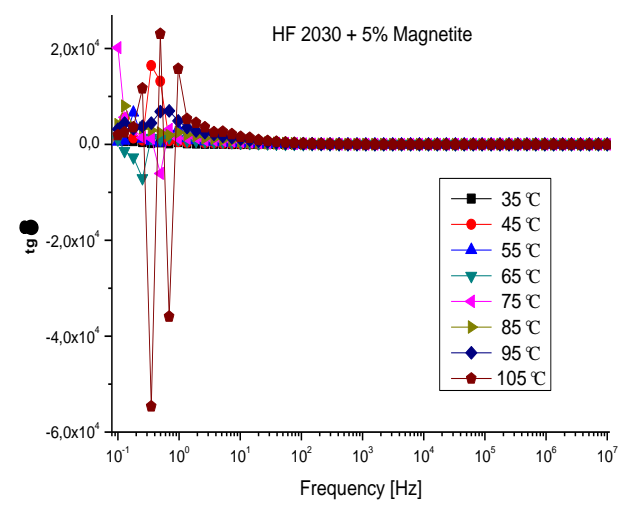

Fig. 12. Frequency dependencies of loss tangent, $\operatorname{tg} \delta$, corresponding to HF $2030+5 \%$ Magnetite

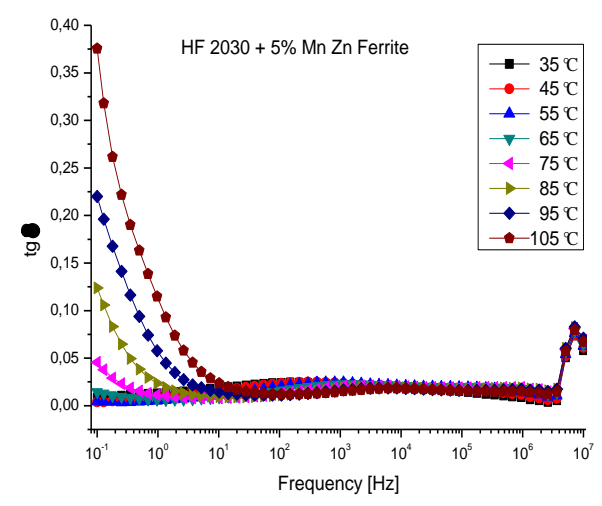

Fig. 13. Frequency dependencies of loss tangent, $\operatorname{tg} \delta$, corresponding to HF $2030+5 \%$ MnZnFerrite

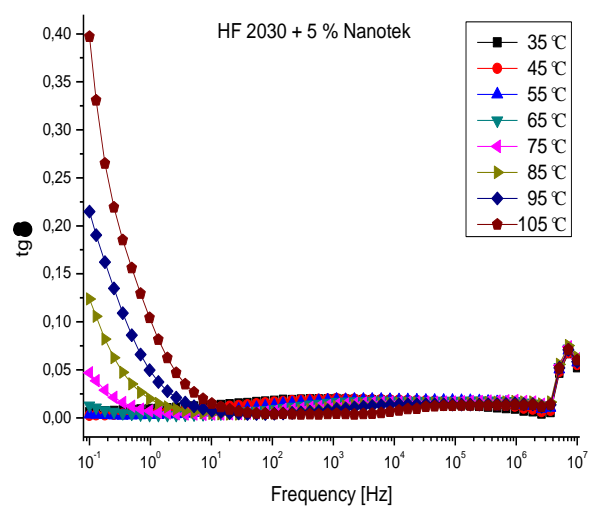

Fig. 14. Frequency dependencies of loss tangent, $\operatorname{tg} \delta$, corresponding to HF $2030+5 \%$ Nanotek 
The tangent loss parameter is related to the ability of the material to be penetrated by an electric field and dissipate energy as heat.

Fig. 10 shows frequency dependencies of loss tangent, $\operatorname{tg} \delta$, corresponding to HF 2030. Fig. 11-15 show the frequency dependencies of loss tangent, $\operatorname{tg} \delta$, corresponding to HF 2030 with $5 \%$ of various nanoparticles.

We observed the remarkable stability of characteristic vs. frequency for entire domain of temperatures, especially for temperatures higher than $80^{\circ} \mathrm{C}$, for the frequency domain of $10^{-1}-10^{7} \mathrm{~Hz}$.

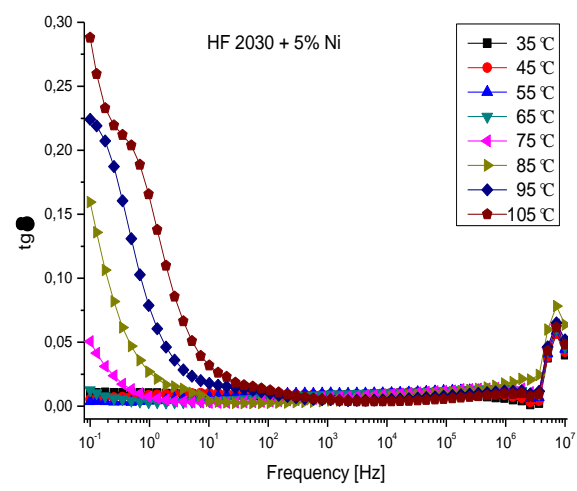

Fig. 15. Frequency dependencies of loss tangent, $\operatorname{tg} \delta$, corresponding to HF $2030+5 \% \mathrm{Ni}$

\section{CONCLUSION}

The innovative bonding process, activated by electromagnetic waves, will have immediate effect on manufacturing industry offering new opportunities in terms of: a) Simplification of productive layout: the disengaging from short open time of hot-melt adhesives will allow the elimination of complex automation systems needed for the rapid application of the adhesives on the substrates. b) More flexible design: the opportunity of externally heating the adhesive by radiofrequency will offer the possibility of applying a solid bead of adhesive onto complex joint substrates unreachable from traditional adhesive dispensing systems. c) Process rapidity: the cycle time of adhesive bonding process will be reduced, making possible a simultaneous assembling process of different elements.

Tthe addition of $5 \%$ magnetite would be a reasonable solution for the proposed composites in association with electromagnetic field, because the dielectric properties, $\varepsilon^{\prime}$ and $\operatorname{tg} \delta$, presented an expected increase with the increase of temperature (Fig. 7, Fig. 12). The dielectric constant, $\varepsilon^{\prime}$, increased from $\left(-2 \times 10^{2)}\right.$ to $\left(6 \times 10^{2)}\right.$ and the loss tangent increased from $\left(-6 \times 10^{4}\right)$ to $\left(2 \times 10^{4}\right)$. The increase of dielectric properties with temperature is due to greater freedom of movement of the dipole molecular chains within the adhesive at high temperature. But the most interesting and unexpected feature was the remarkable stability of characteristic vs. frequency for entire domain of temperatures, especially for temperatures higher than $80^{\circ} \mathrm{C}$

\section{ACKNOWLEDGMENT}

This paper was supported by the Manunet Eranet Magbond 7-042/2011 project financed by Uefiscdi and the project Perform-Era "Postdoctoral Performance for Integration in The European Research Area" (ID-57649), financed by European Social Fund and the Romanian.

\section{REFERENCES}

[1] S. Förster and M. Antonietti, "Amphiphilic block copolymers in structure-controlled nanomaterial hybrids," Advanced Materials, vol. 10, pp. 195-217, 1998.

[2] D. Dunn, Adhesives and Sealants - Technology, Applications and Markets, ed. Smithers Rapra Technology, 2003.

[3] W. E. Webb and R. H. Church. Measurement of dielectric properties of minerals at microwave frequencies. [Online]. Available: http://stacks.cdc.gov/view/cdc/10593/

[4] N. E. Hill, W. F. Vaughn, A. H. iesPrice, and M. Davies, Dielectric Properties and Molecular Behavior Van Nostrand, Reinhold Company, London, UK, 1969.

[5] A. Lu, E. L. Salabas, and F. Schüth, Magnetic Nanoparticles: Synthesis, Protection, Functionalization and Application, 2007.

[6] S. Ayyappan, S. Mahadevan, P. Chandranohan, M. P. Srinivasan, J. Philip, and B. Raj, Influence of $\mathrm{CO}_{2}$ Ion Concentration on the Size, Magnetic Properties and Purity of $\mathrm{CoFe}_{2} \mathrm{O}_{4}$ Spinel Ferrite Nanoparticles, 2010.

[7] Novocontrol guidelines. [Online]. Available: http://www.novocontrol.de.

[8] F. Kremer and A. Schönhals, Broadband Dielectric Spectroscopy, Berlin, 2002.

[9] C. P. Smyth, "Dielectric behavior and Structure," McGraw Hill, New York, 1955, pp. 53.

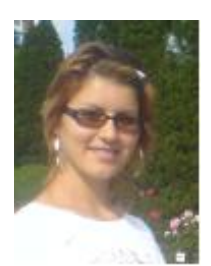

Vasilica Alina Neamtu is a Post $\mathrm{PhD}$ student within Technical University of Iasi, Faculty of Electrical Engineering, Department of Electrical Measurements and Materials. In 2006, respectively 2008, she graduated the bachelor and master courses at the same faculty, and since then she was granted an Erasmus and Titulescu scholarships (France, Spain) and she participated in different research internships and trainings abroad (Italy, Spain). In 2010 she sustained her Ph.D. thesis with the title: "Online systems for industrial quality evaluation and applications". Currently, her research interests are related to: changes in the structure and properties of nanocomposite materials using electromagnetic energy in the microwave range. She is the first author or the co-author of 12 scientific papers published in proceedings of international conferences.

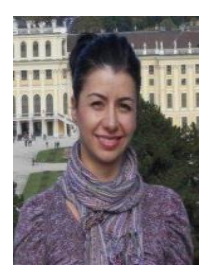

Cristina Bratescu is a research assistant within the Faculty of Electrical Engineering, Department of Electrical Measurements and Materials. In 2006, respectively 2007, she graduated the bachelor and master courses at the same faculty, and since then she was granted an Erasmus and ONBSS scholarships (Spain, France) and she participated in different research internships and trainings abroad (Canada, Turkey, South Korea and Bulgaria). In October 2010 she sustained her Ph.D. thesis with the title „Researches regarding ceramic nanoporous thin films of zeolite crystals for low dielectric constant materials". Currently, her research interests are related to: nanoceramics materials, simulation and modeling of electromagnetic behavior of ceramics materials (QWED and CST Software), low $k$ materials. She is the first author or the co-author of 20 scientific papers published in proceedings of international conferences.

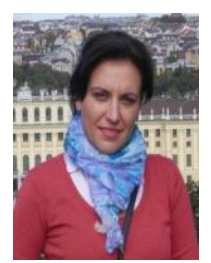

Ramona Burlacu is a research assistant within Technical University of Iasi, Faculty of Electrical Engineering, Department of Electrical Measurements and Materials. In 2006, respectively 2007, she graduated the bachelor and master courses at the same faculty. She participated in different research internships and trainings abroad (Canada, France, South Korea, Spain). In October 2010 she sustained her Ph.D. thesis with the title ,Research on the development of shields and absorbent panels based on nanostructured composites with predefined architecture and electromagnetic properties in GHz". Currently, her research interests are related to: materials for electromagnetic shielding, composite materials, biosensors, dielectric spectroscopy. She is the first author or the co-author of 21 scientific papers published in proceedings of international conferences. 